\title{
Design of Cascaded H Bridge STATCOM with Distributed Control
}

\author{
$\mathrm{He} \mathrm{Li}^{1}$, Yanchi Zhang ${ }^{1, *}$, Da Xie ${ }^{2}$ and Maoye Wang ${ }^{1}$ \\ ${ }^{1}$ School of Electrical Engineering, Shanghai Dianji University, 201306 Shanghai, China \\ ${ }^{2}$ School of Electronic Information and Electrical Engineering, Shanghai Jiaotong University, 200240 Shanghai, China
}

\begin{abstract}
Aiming at the problem of low redundancy in the structure of the cascaded H-bridge STATCOM. This paper studies and designs a cascaded H-bridge STATCOM device based on STM32 distributed control. The article introduces the basic topology of the device and the realization principle of the control system. The turn-on and turn-off time of the MOSFET in each H-bridge is calculated by using the law of minimum harmonics, realize the output of the fitted sine wave. A simulation model of $12 \mathrm{H}$ bridge cascade is built in the EMTP power system electromagnetic transient analysis simulation software. The simulation results show the correctness and reliability of the design.
\end{abstract}

\section{Introduction}

Along with the development of the industry, to the requirement of power quality enhances unceasingly, more and more widely used in power system, flexible ac transmission system (FACTS) for power transmission, reactive and static synchronous compensator (STATCOM) as the core device with fast response speed, good effect of adjusting, operating a wide range, output reactive current is not affected by system voltage of the many advantages[1]. The voltage withstand value of the switching equipment in STATCON has always been an obstacle to the voltage grade upgrade of a reactive power compensation device[2]. In order to obtain multi-level high voltage output by using low voltage withstand switching devices, many methods have been explored. For example, the two-stage pipe clamp multi-level structure, the flyover capacitor clamp multi-level structure and the H-bridge cascade multi-level structure[3]. Among these methods, H-bridge cascade multi-level structure, whose basic power unit is H-bridge with independent DC power supply, is a series structure formed by cascading several H-bridge units, which does not have the problem of DC capacitance and partial voltage[4]. It also has the advantages of easy expansion, high quality output voltage and low harmonic content[5]. Existing cascaded H-bridge multilevel circuits have the following problems in practical application: The power supply of the control system and the trigger system is more complex, requiring multiple groups of isolated power supply, and the requirement of high voltage level. The required tolerance value is the voltage value of the access system. System information transmission wiring is complex, in order to isolate the need in the use of communication level, must choose optical fiber communication, increasing the cost; The cost of IGBT is higher. Main features of this design is to put distribution control system chip STM32 and main control system in the unity of potential, and will trigger the potential part and the power of the power device independence, this solves the important problem of multilevel power need more potential, at the same time satisfy the STM32 power supply reliability, and communication using equipotential, eliminating the high cost of optical fiber communication.

\section{REALIZATION OF BASIC TOPOLOGY AND DISTRIBUTED CONTROL SYSTEM OF CASCADE H-BRIDGE STATCOM DEVICE}

\subsection{The main circuit topology of cascade $H$ bridge STATCOM}

The inverter part of STATCOM main circuit adopts 12 unit $\mathrm{H}$ bridge cascade The advantages of this are as follows:

Switching devices and capacitors with lower voltage resistance value are used to achieve high voltage and large power conversion.

A better sinusoidal waveform is obtained with a lower switching frequency.

It has the advantages of easy expansion, high quality output voltage and low harmonic content.

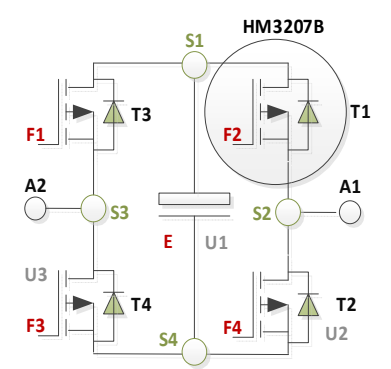

Fig. 1. H bridge element topology

* Corresponding author: zhangyc@sdju.edu.cn 


\subsection{CASCADE H-BRIDGE STATCOM BASIC H- BRIDGE UNIT}

Each H-bridge unit is composed of 4 fully controlled devices and an electrolytic capacitor, as shown in Fig. 1. Each H-bridge unit is equipped with an independent DC power supply, which is realized by full bridge rectifier with three-terminal voltage regulator.

$\mathrm{H}$ bridge in the process of work, the four field effect tube T1, T2, T3, T4, a total of four kinds of working state $(\mathrm{T} 1, \mathrm{~T} 2, \mathrm{~T} 3, \mathrm{~T} 4)$, namely, $(1,0,0,1)(0,1,0,1),(0$, $1,1,0),(0,1,0,1)$, including $\mathrm{T} 1$ or $\mathrm{T} 2, \mathrm{~T} 3, \mathrm{~T} 4)=1$ said $\mathrm{T} 1$ or $\mathrm{T} 2, \mathrm{~T} 3, \mathrm{~T} 4)$ conduction, $\mathrm{T} 1$ or $\mathrm{T} 2, \mathrm{~T} 3, \mathrm{~T} 4)=0$ means $\mathrm{T} 1$ or $\mathrm{T} 2, \mathrm{~T} 3, \mathrm{~T} 4)$ shut off, because the T1 and T2, $\mathrm{T} 3$ and T4 conduction device will cause short circuit at the same time, Therefore, the chip in the device should avoid triggering two power field effect tubes on the same bridge arm. The working states described in the above four are respectively discussed below:

- State $1,(\mathrm{~T} 1, \mathrm{~T} 2, \mathrm{~T} 3, \mathrm{~T} 4)=(1,0,0,1)$

Switch tubes T1 and T4 on and T3 and T2 off. The working process of the circuit is shown in Figure 3(a). The output voltage of A1A2 port is $+\mathrm{E}$.

- State 2, $(\mathrm{T} 1, \mathrm{~T} 2, \mathrm{~T} 3, \mathrm{~T} 4)=(1,0,1,0)$

Switches T1 and T3 are on and T2 and T4 are off. The working process of the circuit is shown in Fig. 3(b). The output voltage of port A1A2 is 0 .

- State 3, $(\mathrm{T} 1, \mathrm{~T} 2, \mathrm{~T} 3, \mathrm{~T} 4)=(0,1,1,0)$

Switch tubes T2 and T3 are on and T1 and T4 are off. The working process of the circuit is shown in Fig. 3(c). The output voltage of port A1A2 is -E.

- $\quad$ state $4,(\mathrm{~T} 1, \mathrm{~T} 2, \mathrm{~T} 3, \mathrm{~T} 4)=(0,1,0,1)$

Switch tubes $\mathrm{T} 2$ and $\mathrm{T} 4$ are on and $\mathrm{T} 1$ and $\mathrm{T} 3$ are off. The working process of the circuit is shown in Fig. 3(d). The output voltage of port A1A2 is 0 .

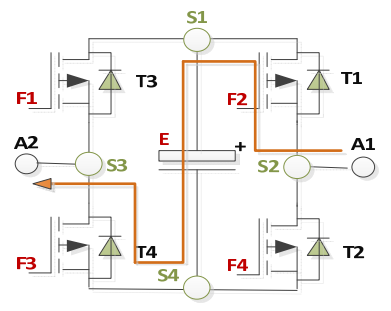

(a) Working state 1

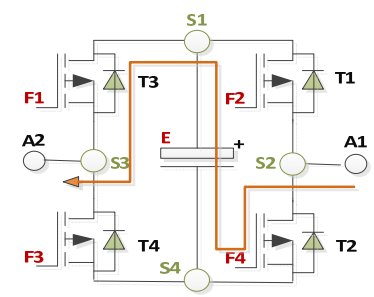

(c) Working state 3

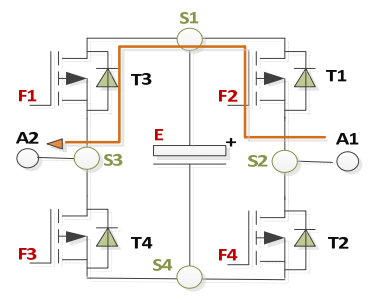

(b) Working state 2

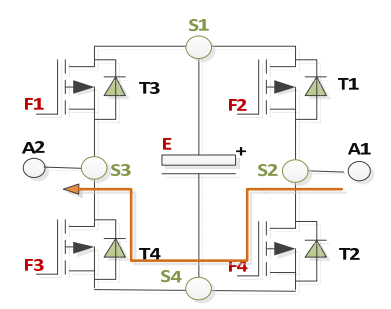

(d) Working state 4
Fig.2. Operating state of single H-bridge in continuous conduction mode

\subsection{Implementation of STM32 distributed control system}

The control part of the device is composed of the main engine and the slave unit. United by sending information, focusing on DSP chip TMS320F28335 as instruction, the machine is also distributed control section from stmicroelectronics company has powerful ability of control and signal processing STM32F103C8T6 chip, the chip has a 32-bit arm-based core with $64 \mathrm{~KB}$ flash microcontroller, with two 12-bit A/D converter, up to 16 input channels, at the same time it has 7 timer, 9 communication interface. This system has designed the following functions.

- Trigger part.

- The collection and monitoring of $\mathrm{H}$ bridge's working state.

- Communication.

- Trigger the power supply.

\section{THE WORKING PRINCIPLE AND MODULATION STRATEGY OF CASCADE H BRIDGE STATCOM DEVICE}

\subsection{STATCOM compensation voltage and power Angle calculation}

To determine the amplitude and phase of the voltage emitted by STATCOM. The compensating voltage and Angle of STATCOM will be calculated below. In the following formula, $\mathrm{U} 1$ and $\mathrm{U} 2$ are the amplitude of STATCOM voltage and the amplitude of system voltage respectively.

Current calculation:

$$
\begin{aligned}
I & =\frac{\dot{U}_{1}-\dot{U}_{2}}{j X_{L}}=\frac{U_{1} \cos \delta+j U_{1} \sin \delta-U_{2}}{j X_{L}} \\
& =\frac{U_{1} \sin \delta+j\left(U_{2}-U_{1} \cos \delta\right)}{X_{L}}
\end{aligned}
$$

Power calculation: $P_{1}=P_{2}$

$S_{1}=\dot{U}_{1} \cdot \dot{I}=\left(U_{1} \cos \delta+j U_{1} \sin \delta\right) \cdot \frac{U_{1} \sin \delta+j\left(U_{2}-U_{1} \cos \delta\right)}{X_{L}}$

$=\frac{U_{1} U_{2} \sin \delta}{X_{L}}+j \frac{U_{1}^{2}-U_{1} U_{2} \cos \delta}{X_{L}}$

$=P_{1}+j Q_{1}$

Among them: Active power

$$
P_{1}=\frac{U_{1} U_{2} \sin \delta}{X_{L}}
$$

Reactive power

$$
Q_{1}=\frac{U_{1}^{2}-U_{1} U_{2}}{X_{L}}
$$

For STATCOM, $P_{1}<Q_{1}$, so $\delta \rightarrow 0$

So there's a formula $\sin \delta \approx \delta, \cos \delta \approx 1$

Thus the power can be simplified as 


$$
\begin{aligned}
& P_{1}=\frac{U_{1} U_{2} \delta}{X_{L}} \\
& Q_{1}=\frac{U_{1}^{2}-U_{1} U_{2}}{X_{L}}
\end{aligned}
$$

For a compensation system, $\mathrm{Q}$ is the load reactive power and is a determined value, then the voltage can be calculated.

$$
U_{1}=\frac{U_{2} \pm \sqrt{U_{2}^{2}+4 Q_{1} X_{L}}}{2} \quad\left(U_{1}>0\right)
$$

In Equation (6), when $Q_{1}>0$ Take a minus sign, $Q_{1}<0$, Take a plus sign.

$$
\delta=\frac{P_{1} X_{L}}{U_{1} U_{2}}
$$

The amplitude of U1 is determined by the synthesis of several step waves of the inverter voltage.

The relationship between the inverter voltage and the system voltage is shown in Fig. 3 .

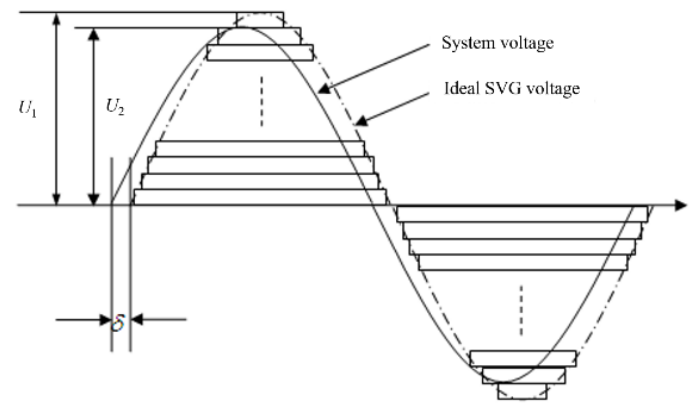

Fig.3. Relationship between inverter voltage and system voltage

\subsection{Inverter modulation strategy in STATCOM device}

The control strategy of STATCOM determines the excellent degree of its function, and the modulation strategy of the inverter determines the output performance of the inverter, and then determines the performance of STATCOM. The device uses the principle of minimum harmonic method to realize the function. The off-line calculation of the principle of minimum harmonic method is simple, and the output waveform characteristics are good.

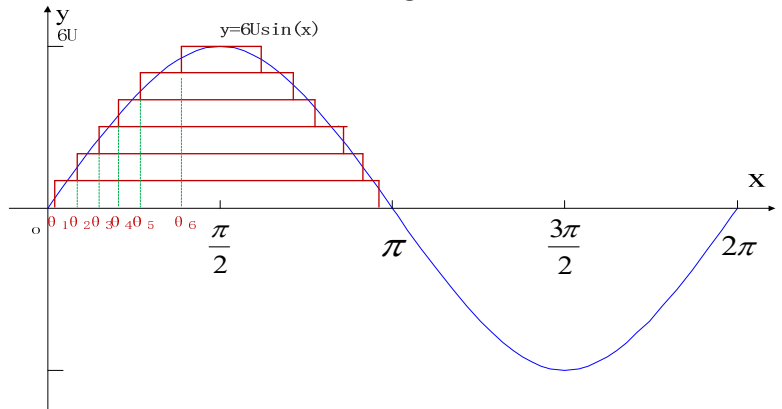

Fig.4. Schematic diagram of 12 link step wave approximating sine wave
Fig. 4 is the schematic diagram of step wave approximating sine wave. The calculation of $\theta_{1}, \theta_{2}, \theta_{3}$, $\theta_{4}, \theta_{5}, \theta_{6}$ is realized in accordance with the principle of least low-order harmonics. Therefore, it is stipulated that the stepped waveform area of output of each part is equal to the corresponding sine wave area respectively. Take the ith block $(I=1,2,3,4,5,6)$, so

$\int_{\theta_{i-1}^{\prime}}^{\theta_{i}}[6 U \sin (x)-(i-1) U] d x=\int_{\theta_{i}}^{\theta_{i}^{\prime}}[i U-6 U \sin (x)] d x$

$\theta_{i-1}^{\prime}=\arcsin [(i-1) U], \quad \theta_{i}^{\prime}=\arcsin (i U)$

$\mathrm{U} 1$ can be obtained by the number of $\mathrm{H}$ Bridges in series and the voltage.

Solve the integral formula, and we can get:

$$
\theta_{i}=i \theta_{i}^{\prime}-(i-1) \theta_{i-1}^{\prime}+6 \cos \theta_{i}^{\prime}-6 \cos \theta_{i-1}^{\prime}
$$

According to the above analysis and calculation, the turn on and off time of MOSFET on each $\mathrm{H}$ bridge can be obtained, so as to fit the sine wave output, complete the DC/AC function, and then realize the function of STATCOM device.

\section{THE SIMULATION RESEARCH}

Using EMTP power system electromagnetic transient analysis simulation software, a $12 \mathrm{H}$ bridge cascade STATCOM device model is built for simulation analysis. The parameters set in the simulation model are as follows: rated power 1MVAR, STATCOM output voltage $10 \mathrm{kV} / 50 \mathrm{~Hz}$, rated current $100 \mathrm{~A}$, DC side rated voltage $800 \mathrm{~V}$, DC side capacitance $6800 \mu \mathrm{F}$, and the number of one-phase chain 12.

Figure 5 shows the voltage waveform at the DC side of STATCOM device, namely the voltage waveform at both ends of Bridge H. It can be seen from the figure that the voltage is basically stable at $800 \mathrm{~V}$, the deviation is no more than $5 \%$, and there is no imbalance. Figure 6 shows the waveform of A-phase output voltage and output current. The output waveform is trapezoidal wave fitting sinusoidal wave output, which is in line with the control strategy.

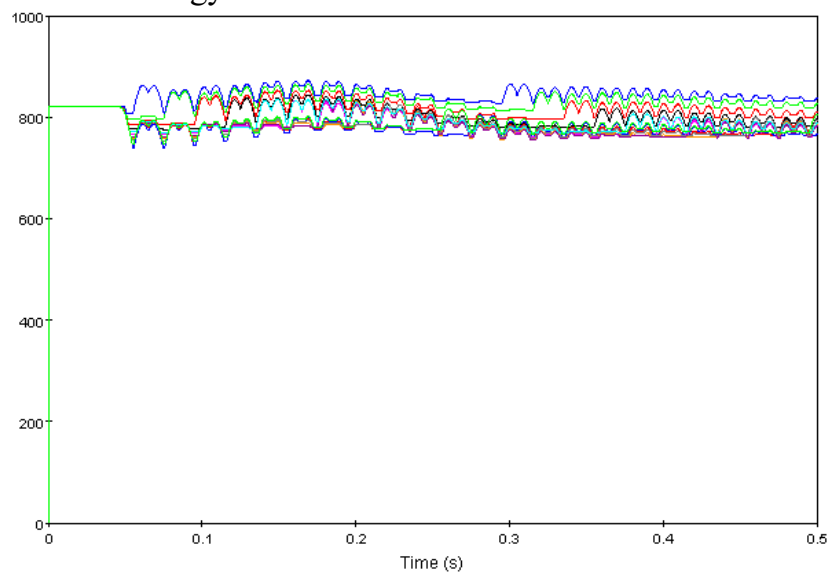

Fig.5. DC side voltage waveform of STATCOM device 

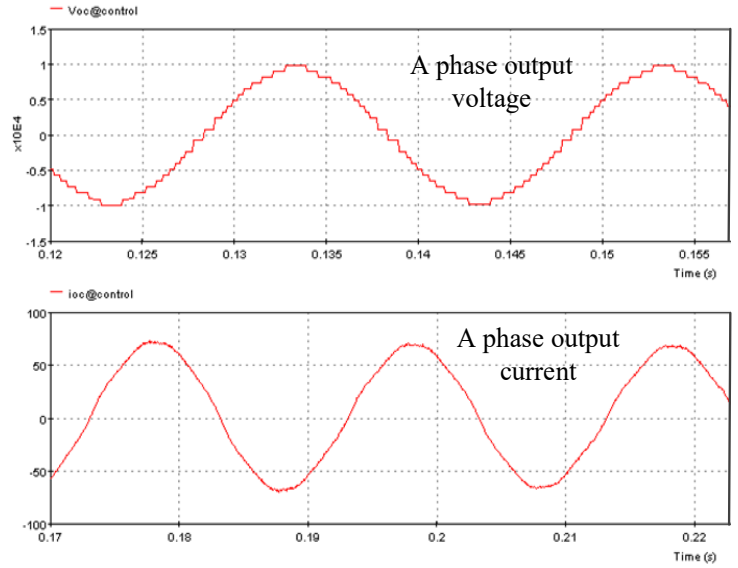

Fig.6. Simulation waveform of A-phase output voltage and current

The simulation waveform of the output voltage and current of three-phase A, B and C is shown in Figure 7. The three-phase output voltage conforms to the principle of multi-overlapping addition and is formed by the superposition of stepped waves. The sinusoidal wave output is fitted, and the three-phase current is also output in the form of sine. Figure 8 shows the active and reactive power curves of STATCOM device, which shows that the device effectively compensates the reactive power emitted by the load.
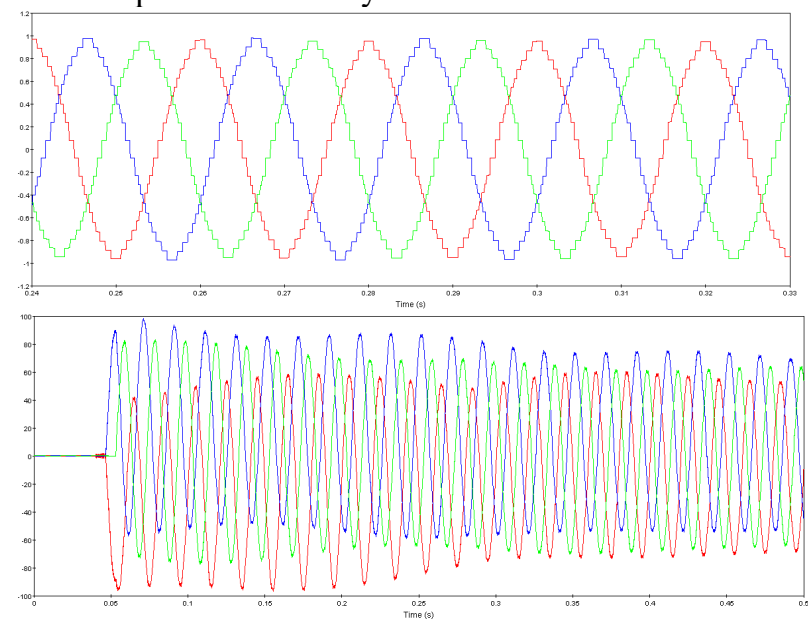

Fig.7. Output voltage and current simulation waveform of three phases A, B and C

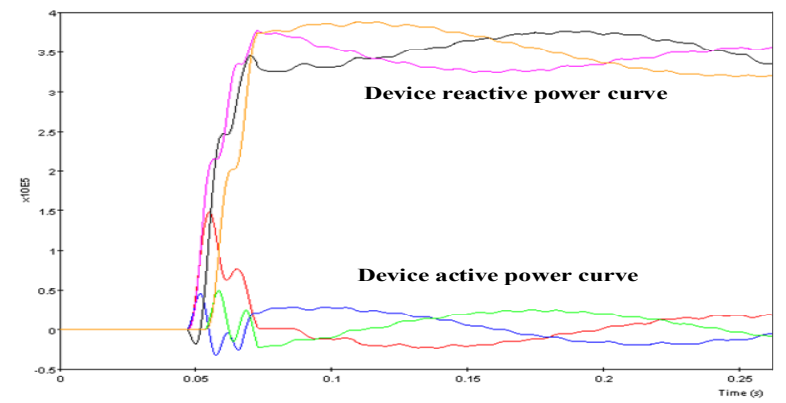

Fig.8. Active and reactive power curves of STATCOM device

\section{CONCLUSION}

This paper studies and designs a cascade H-bridge STATCOM device based on STM32 distributed control. The device realizes multi-level power supply and only needs one potential. Under the control of the minimum harmonic law, the step wave superposition is realized and the sinusoidal output is fitted, which provides a method for dynamic reactive power compensation. In addition, due to the use of slave distributed control, host unified scheduling, to meet the control precision and reliability greatly improved. In addition, the communication achieves equal potential, which saves a lot of cost compared with optical fiber communication. The analysis results show that the theoretical analysis is consistent with the simulation results, which verifies the correctness of the theoretical analysis and the possibility of practical application.

\section{Acknowledgments}

The research in this paper was supported by Research on Energy Systems of Smart Park. Research and Demonstration on Key Technologies of Multi-functional Energy Router of Shanghai Science and Technology Commission (18DZ1203700).

\section{References}

1. Hatano $\mathrm{N}$, Ise $\mathrm{T}$. A configuration and control method of cascade H-bridge STATCOM[C]// Power and Energy Society General Meeting - Conversion and Delivery of Electrical Energy in the, Century. IEEE, 2008:1-8.

2. Ying $\mathrm{C}$, Qiang $\mathrm{G}, \mathrm{Xu} \mathrm{D}$. Control and performance of a medium-voltage cascade H-bridge STATCOM[C]// Power Electronics and Motion Control Conference. IEEE, 2012:2995-2999.

3. Busarello T, Mortezaei A, Morales-Paredes H, et al. Simplified Small-Signal Model for Output Voltage Control of Asymmetric Cascaded H-Bridge Multilevel Inverter[J]. IEEE Transactions on Power Electronics, 2017.

4. Zhang $\mathrm{C}$, Geng $\mathrm{H}$, Li $\mathrm{S}$, et al. Hybrid communication topology and protocol for distributed-controlled cascade H-bridge multilevel STATCOM $[\mathrm{C}] / /$ International Conference on Electrical Machines and Systems. IEEE, 2015:597602.

5. Reddy C L, Kumar P S, Sushama M, et al. A five level cascaded H-bridge multilevel STATCOM[C]// Microelectronics and Electronics. IEEE, 2016:36-41. 